Sección central

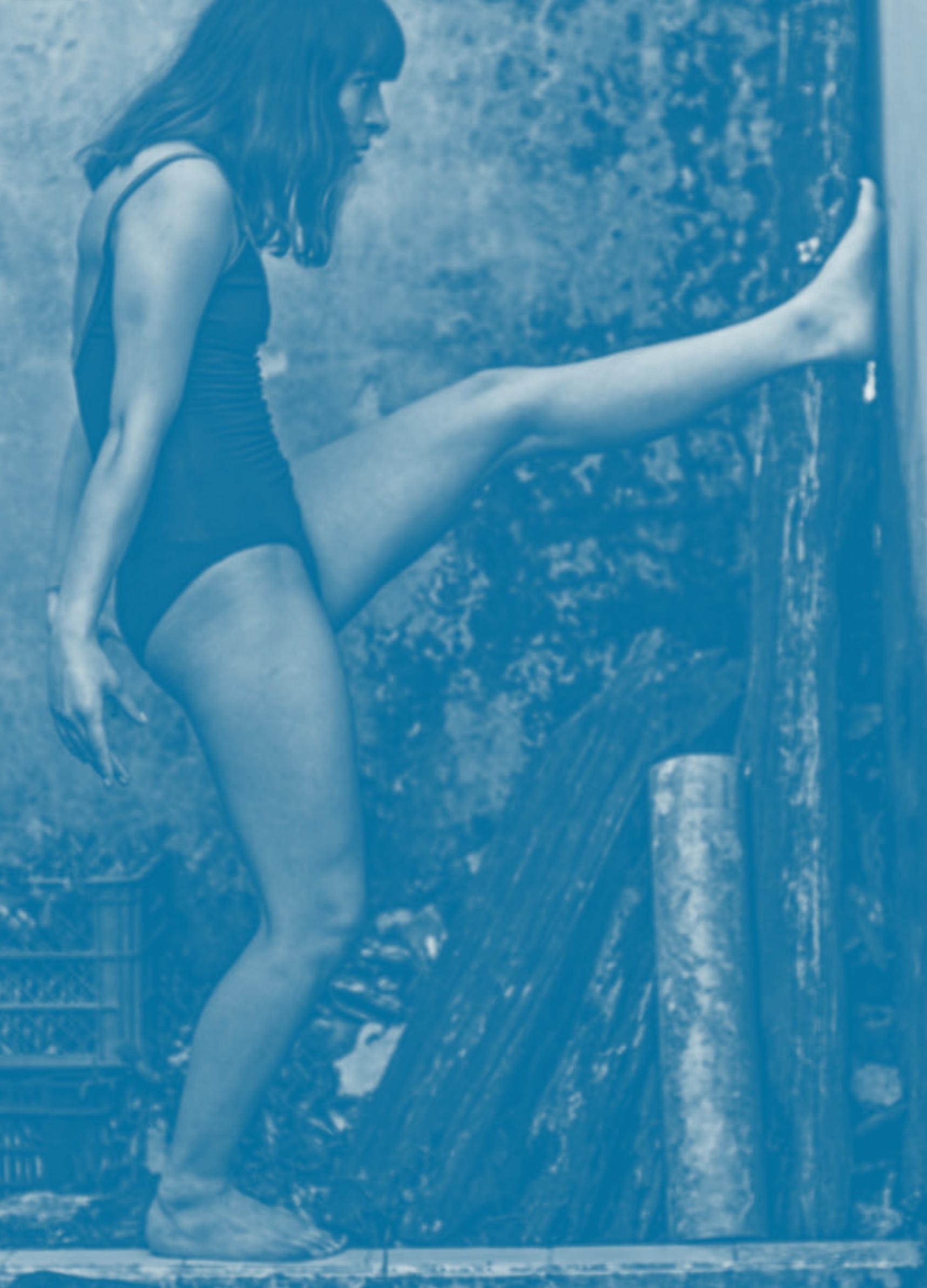




\section{Movimiento auténtico, una aproximación visual en tiempo de confinamiento}

\author{
Ensayo visual \\ Recibido: 20 de junio de 2020 \\ Aprobado: 18 de octubre de 2020 \\ Juana Valentina Jerez \\ Universidad Distrital Francisco José de Caldas, \\ Colombia \\ phiuanavalentina@gmail.com
}

Cómo citar este artículo: Jerez, J. (2021). Movimiento auténtico, una aproximación visual en tiempo de confinamiento. Estudios Artísticos: revista de investigación creadora, 7(11) pp. 308-324. DOI: https://doi.org/10.14483/25009311.17561

\begin{abstract}
Resumen
Este ensayo visual presenta la serie Paz sin dueño, en la que con la artista de la danza Carol López, se penetra en una serie de premisas a medida que la bailarina logra plasmar en sus movimientos memorias de su día a día, y las sensaciones que emanan de la experiencia de confinamiento, además de los momentos relacionados con sus hábitos y transcurir por la ciudad. Esta es una serie fotográfica que rescata visualmente parte del proceso del movimiento auténtico, corre el velo de lo corpóreo y profundiza en el proceso de presentar físicamente el diseño en movimiento.
\end{abstract}

\section{Palabras claves}

Cuerpo; movimiento; visualidad; fotografía

$<$

De la serie Paz sin dueño (2020). Juana Valentina Jerez.

Fotografía analógica. 
Authentic Movement, a Visual Approach in a Time of Confinement

\begin{abstract}
This visual essay presents the series Paz sin dueño, in which, along with dance artist Carol López a series of premises are examined, as the dancer manages to capture in her movements memories of her day to day, and the sensations that emanate from the confinement experience, in addition to the moments related to her habits and dwelling in the city. This is a photographic series that visually rescues part of the authentic process of movement, lifts the veil of the corporeal and delves into the process of physically presenting design in movement.
\end{abstract}

\section{Keywords}

Body; movement; visuality; photography

\section{Mouvement authentique, une approche visuelle en temps de confinement}

\section{Résumé}

Cet essai visuel présente la série Paz sin dueño, dans laquelle, avec l'artiste de la danse Carol López, une série de prémisses sont examinées. La danseuse parvient à capturer dans ses mouvements des souvenirs de son quotidien et des sensations qui émanent de l'expérience d'enfermement, en plus des moments liés à ses habitudes et à sa vie en ville. Il s'agit d'une série photographique qui sauve visuellement une partie du processus authentique du mouvement, lève le voile du corporel et plonge dans le processus de présentation physique du design en mouvement.

\section{Mots clés}

Corps ; mouvement ; visualisation ; photographie

\section{Movimento autêntico, uma aproximação visual em tempo de confinamento}

\section{Resumo}

Este ensaio visual apresenta a série Paz sin dueño, na qual conjuntamente com a artista da dança Carol López, se adentra a uma série de premissas nas quais se entra a medida em que ela consegue colocar em seus movimentos memórias do seu dia a dia, das sensações que, neste caso, se relacionam com a experiência de confinamento; além disso, as lembranças relacionadas com o que foi seu hábito e transcorrer na cidade. Esta, é uma série fotográfica que resgata visualmente parte do processo do movimento autêntico, faz uma incisão no corpóreo e no processo de apresentar fisicamente o desenho em movimento.

\section{Palavras chave}

Corpo; movimento; visualidade; fotografia

\section{Nukakin kllurispa mailla ukupi kaskata kawaspa}

\section{Maillallachiska:}

Kai mailla kawachiku kilkai suti allilla kai mana sapalla kidai ruraska kai runa danzaría Carol López, suti pai munaku puncha puncha apachinga paikuna iachakuskatasgknata llalichingapa kai ruraikuna kami kawaspa iuiarispa Nukanchipa kausai pangapi ruraska kunauramanda chasallata ñugpamanda.

\section{Rimangapa Ministidukuna:}

Nukanchi kikin, kullirii, kai, fotokuno surkui

La reavivación del cuerpo desde una condición cuasi obligada de quietud implica una decisión individual. Estos espacios de creación colectiva dependen de las circunstancias en que se da; en nuestro caso, un grito de amparo entre las implicaciones de la experiencia de lo pasajero que precisaban ser subsanadas a través de la meditación en movimiento ${ }^{1}$ como motor creativo. Estas dejan como circunstancia la captura de la búsqueda de compresión, la imaginación en torno al movimiento y a su experiencia. El cuerpo experimenta con la sensibilidad. Se permite estar sin estar, viaja desde lo más cercano de su memoria a lo que considera familiar para alinear, recaer, dispersar. Ella misma, atenta e incorrecta, enmarca sutilmente el regreso, elimina pensamientos y trae movimientos, se va y luego retorna.

Parte de la creación de este momento queda el propósito de generar desde una perspectiva visual una aproximación a esta práctica corporal que 
suscita una coreografía espacial la cual rescata la perdida del uso del cuerpo en tiempos de confinamiento; un cuerpo que se configura como un cuerpo innecesario e incapaz, el cual tiene la oportunidad de volver.

El hombre lo vive todo a la primera y sin preparación. Como si un actor representase su obra sin ningún tipo de ensayo

(Milan Kundera 1984).

Es suficiente el ahora y como presente libera, contiene, respira, sueña, encuentra su paz y no tiene dueño. Es el lugar preciso donde se dialoga con el espacio y se diseña influenciada por la afectividad de su transcurso en lo cotidiano. Pero esta sensibilidad corpórea cae en el encierro obligado; sin embargo, ese mundo que conoce le da la capacidad de transfigurar su creación partiendo de la intuición. A partir de ahí se permite asumir su existencia en el transitar de los días, se impulsa a vivir en medio de ansiedades y corduras, quiebra y reedifica, sintoniza su entorno y vuelve todo un solo ambiente. La oscuridad entre sus ojos y el aire que incita le acompañan cuidadosamente en cada parte apropiándose del ritmo, pero su danza es inconscientemente coreográfica.

El cuerpo piensa con todos sus sentidos. Y lo hace de la misma manera que conecta todas las articulaciones; unas más tensas, relajadas otras, distendidas algunas, pero ninguna disfuncional. El cuerpo no es la envoltura del alma. Es nada más que la convoltura de un palimpsesto escrito con plumas, con cinceles y palabras sobre la carne palpitante de una bailarina. Ya quisieran los dioses tener la apariencia de la finitud y la experiencia de lo pasajero; no del tiempo que pasa, sino de lo pasajeros que somos en el tiempo. Y por eso, porque somos pasajeros es que se nos da el acceso la experiencia de varios tiempos, de todos los tiempos, incluyendo la ilusión de un tiempo expandido en la eternidad. La amplia gamma de los tiempos se parece a la escalera de grises de una imagen en blanco y negro. Una imagen que esconde en medio de sus transiciones todos los colores del mundo y a veces oculta un tono tan oscuro que nos seduce a tocarlo, pero al volvernos transgresores, nos toca pagar un precio equivalente a la pérdida de la visión. ${ }^{2}$

Paz sin dueño es entonces una poética visual que capta el retorno a nuestro cuerpo a través de la danza como herramienta creativa. Al desarrollar esta experiencia se resalta la importancia de la relación entre interprete y fotógrafo, ya que al llegar a este lugar de apreciación y creación corporal se rescatan recuerdos de la práctica a la que nos inmiscuimos desde la improvisación en danza, y que nace de mi interés hacía el reconocimiento corporal como artista plástica y visual siendo necesario el ampliar las posturas que sugieren un descubrimiento del cuerpo como revelación del propio ser (Bravo \& Villagrán, 2016).

1 Juan González, Maestro en Artes Escénicas con énfasis en Danza Contemporánea, de la Facultad de Artes ASAB, Universidad Distrital FJC. Magister interdisciplinar en Teatro y Artes Vivas Universidad Nacional de Colombia. Nos presentó el "Autenthic Movement" o "Movimiento Autentico" como técnica para la creación desde el movimiento autodirigido. Además, como herramienta de auto conocimiento y auto consciencia de nuestro cuerpo, desde el espacio de improvisación en danza. Su preparación desde el campo teatral, danzario y performativo, le han llevado a centrar su atención sobre la construcción de la dramaturgia del cuerpo en la escena contemporánea, trabajando en instituciones como la Universidad Distrital en la Facultad de Artes ASAB en el programa de arte danzario para las profundizaciones de DanzaTeatro y composición coreográfica.

2 Este fragmento fue escrito por un observador de la serie quien compartió conmigo su experiencia al ver la serie fotográfica. 



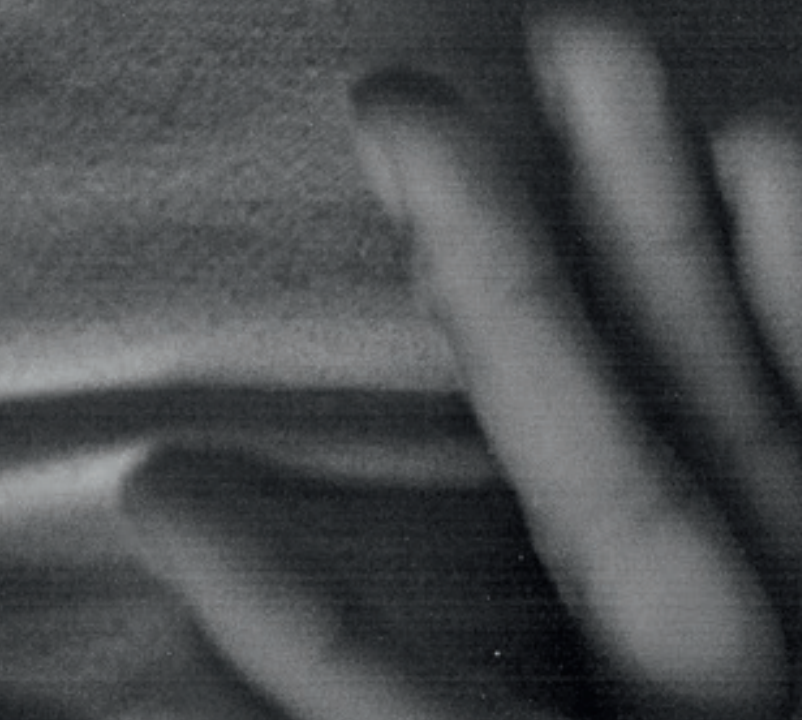


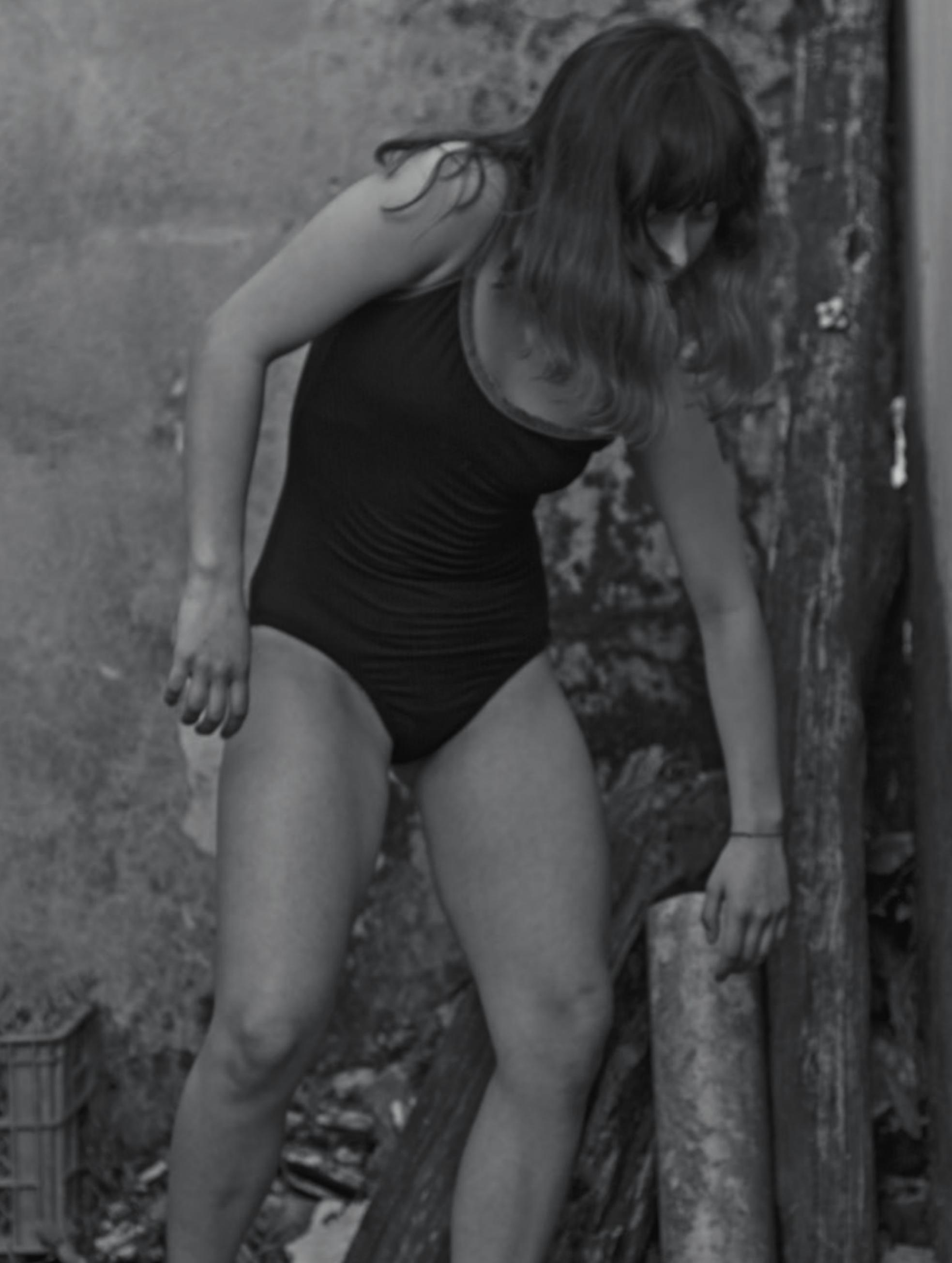





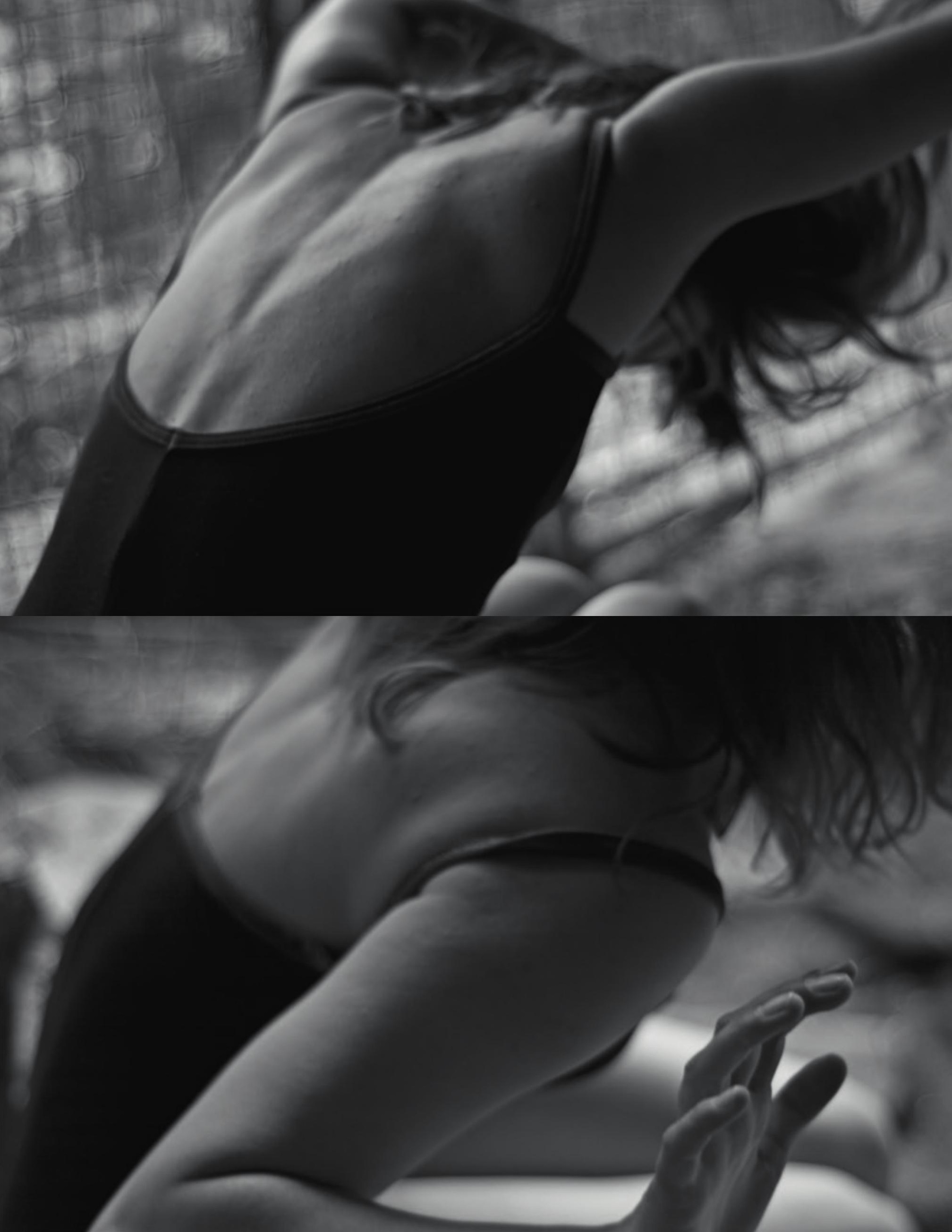




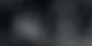




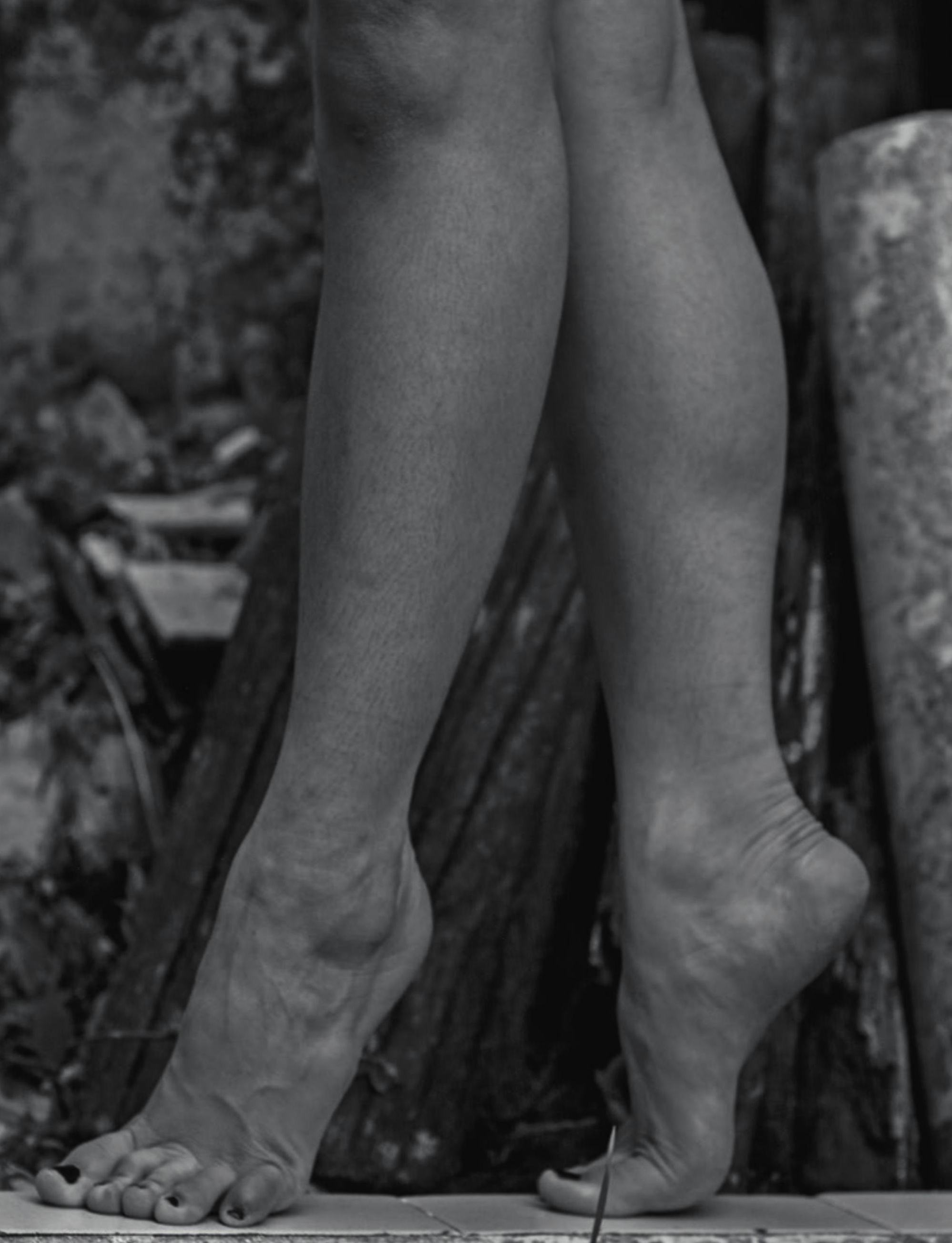




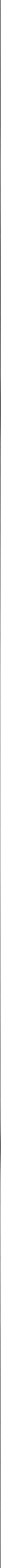




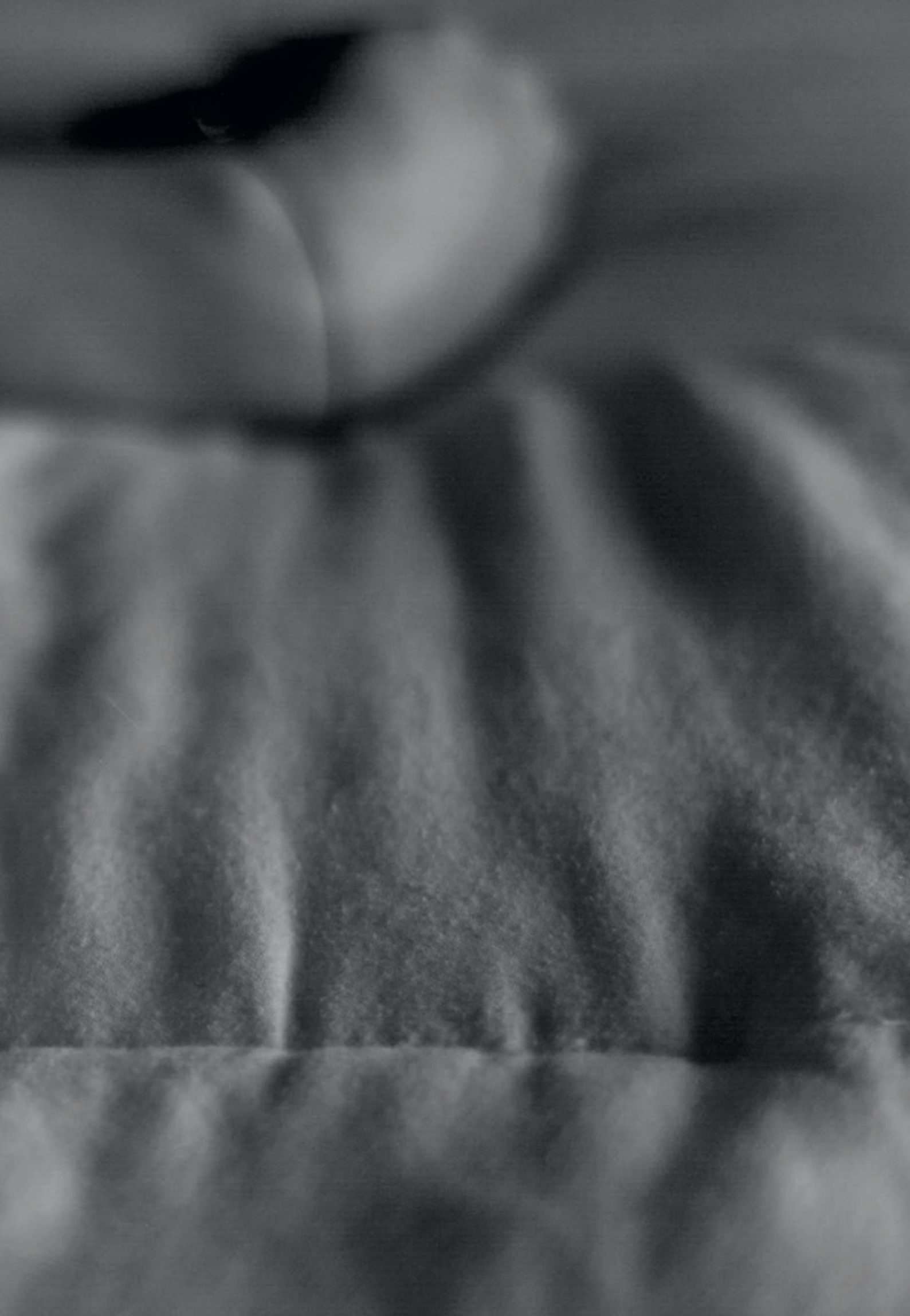





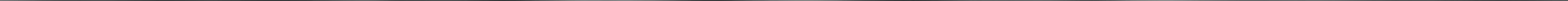




\section{Referencias}

Authentic Movement Community. (s. f.). About Authentic Movement. Authentic Movement Community. Recuperado agosto de 2020, de http://authenticmovementcommunity.org/ about

Castles, J. (2018). Poéticas blandas: ondulantes, sinuosas y distorsiones (2006-2017). Calle 14 Revista De investigación En El Campo Del Arte, 13(23), 16-37. https://doi. org/10.14483/21450706.12986

Gómez Moreno, P. P. (2020). El ensayo visual: una tipología emergente de artículos investigación-creación. Calle 14 Revista De investigación En El Campo Del Arte, 15(28), 221-223. https:// doi.org/10.14483/21450706.16901

Le Breton, David. Cuerpo Sensible / David Le Breton; edición y traducción Alejandro Madrid Zan.. Santiago de Chile: Ediciones Metales Pesados, 2005

Londoño Venegas, S. (2018). El arte de la memoria en la arquitectura. Rogelio Salmona y sus memorias de África: concierto de recuerdos, experiencias y composición. Calle 14 Revista De investigación En El Campo Del Arte, 13(23), 74-100. https://doi.org/10.14483/21450706.12990

Mignolo, W. (2019). Reconstitución epistémica/estética: la aesthesis decolonial una década después. Calle 14 Revista De investigación En El Campo Del Arte, 14(25), 1433. https://doi. org/10.14483/21450706.14132
Ospina Moreno, D. (2019). Sísifos... una montaña en las espaldas, anhelos de una chispa dorada. Calle 14 Revista De investigación En El Campo Del Arte, 14(25), 34-57. https://doi. org/10.14483/21450706.14074

Ramone, H. (2018). De la serie El jardín perdido. Calle 14 Revista De investigación En El Campo Del Arte, 13(24), 288-303. https:// doi.org/10.14483/21450706.13526

Rengifo, S., \& Tsanakas, K. (2020). City of refuge (Ciudad de refugio). Estudios Artísticos, 6(8), 20-41. https://doi. org/10.14483/25009311.15689

Rengifo, S., \& Escobar Neira, F. (2018). El genio secreto del catalejo (Kikkertens Hemmelighedsfulde Aand). Calle 14 Revista De investigación En El Campo Del Arte, 13(24), 254 287. https://doi.org/10.14483/21450706.13525

Rey, S. R. (2019). En el planeta Ingmar Bergman. Estudios Artísticos, 5(6), 30-43. https://doi.org/10.14483/25009311.14098

Roda Fornaguera, M. (2015). Sobre la serie "tiempos revueltos". Estudios Artísticos, 1(1), 156-173. https://doi. org/10.14483/25009311.10253

Rueda Fajardo, S., \& Eljaiek, A. (2016). Escritores, fotografías y retratos; una entrevista a Abdú Eliaiek (3 de febrero 2005). Estudios Artísticos, 2(2), 132-155. https://doi. org/10.14483/25009311.11532 